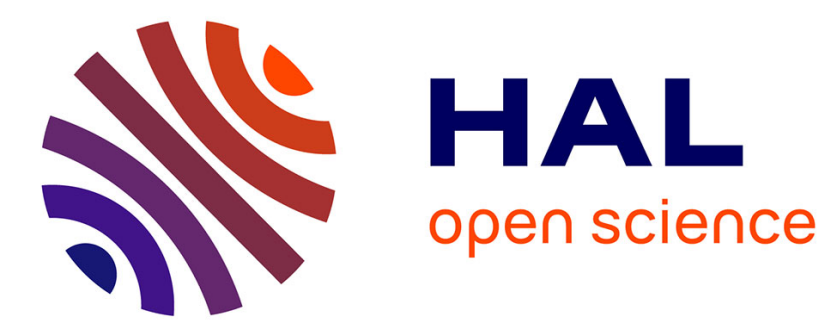

\title{
Melting Curve and Liquid Structure of Nitrogen Probed by X-ray Diffraction to 120 GPa
}

\author{
Gunnar Weck, Frédéric Datchi, Gaston Garbarino, Sandra Ninet, J. A. \\ Queyroux, Thomas Plisson, Mohamed Mezouard, P. Loubeyre
}

\section{- To cite this version:}

Gunnar Weck, Frédéric Datchi, Gaston Garbarino, Sandra Ninet, J. A. Queyroux, et al.. Melting Curve and Liquid Structure of Nitrogen Probed by X-ray Diffraction to 120 GPa. Physical Review Letters, 2017, 119, 10.1103/physrevlett.119.235701 . hal-03017162

\section{HAL Id: hal-03017162 https://hal.science/hal-03017162}

Submitted on 20 Nov 2020

HAL is a multi-disciplinary open access archive for the deposit and dissemination of scientific research documents, whether they are published or not. The documents may come from teaching and research institutions in France or abroad, or from public or private research centers.
L'archive ouverte pluridisciplinaire HAL, est destinée au dépôt et à la diffusion de documents scientifiques de niveau recherche, publiés ou non, émanant des établissements d'enseignement et de recherche français ou étrangers, des laboratoires publics ou privés. 


\title{
Melting Curve and Liquid Structure of Nitrogen Probed by X-ray Diffraction to $120 \mathrm{GPa}$
}

\author{
Gunnar Weck, ${ }^{1}$ Frédéric Datchi, ${ }^{2}$ Gaston Garbarino, ${ }^{3}$ Sandra Ninet, ${ }^{2}$ Jean-Antoine Queyroux, ${ }^{2}$ \\ Thomas Plisson, ${ }^{1}$ Mohamed Mezouar, ${ }^{3}$ and Paul Loubeyre ${ }^{1}$ \\ ${ }^{1} C E A, D A M, D I F$, F-91297 Arpajon, France \\ ${ }^{2}$ Institut de Minéralogie, de Physique des Milieux Condensés et de Cosmochimie (IMPMC), \\ Sorbonne Universités-UPMC Univ. Paris 6, CNRS UMR 7590, IRD UMR 206, MNHN, \\ 4 place Jussieu, F-75005 Paris, France \\ ${ }^{3}$ European Synchrotron Radiation Facility, 71, avenue des Martyrs, CS 40220, 38043 Grenoble Cedex 9, France
}

(Received 29 September 2017; published 4 December 2017)

\begin{abstract}
Synchrotron x-ray diffraction measurements of nitrogen are performed up to $120 \mathrm{GPa}$ to determine the melting curve and the structural changes of the solid and liquid phases along it. The melting temperature exhibits a monotonic increase up to the triple point where the epsilon molecular solid, the cubic gauche covalent solid, and the fluid meet at $116 \mathrm{GPa}, 2080 \mathrm{~K}$. Above, the stability of the cubic gauche phase induces a sharp increase of the melting curve. The structural data on liquid nitrogen show that the latter remains molecular over the whole probed domain, which contradicts the prediction of a liquid-liquid transition at $88 \mathrm{GPa}, 2000 \mathrm{~K}$. These findings thus largely revisit the phase diagram of hot dense nitrogen and challenge the current understanding of this model system.
\end{abstract}

DOI: 10.1103/PhysRevLett.119.235701

The triple bond of the nitrogen molecule is one of the strongest and most stable chemical bonds in nature. This exceptional stability persists under very large compression above $100 \mathrm{GPa}$ in the solid phase. A further increase in density, however, eventually destabilizes the triple bond and provokes a transition to a semiconducting amorphous solid above $150 \mathrm{GPa}$ at room temperature $[1,2]$ or to the crystalline cubic gauche (cg-N) structure at $110 \mathrm{GPa}$ and temperatures above $2000 \mathrm{~K}$ [3]. Another extended crystal was recently discovered above $126 \mathrm{GPa}$ [4]. In the fluid, shock-wave experiments reported that dissociation starts above $30 \mathrm{GPa}, 6000 \mathrm{~K}$ and that a fully dissociated metallic fluid is obtained at about $120 \mathrm{GPa}[5,6]$. Current efforts aim at disclosing the incidence of molecular dissociation in the region of the phase diagram close to the melting curve. Remarkable features are predicted by first-principles simulations such as a maximum on the melting curve, a discontinuity in the melting slope, and a liquid-liquid first order transition $[7,8]$. Up to now, the only experimental informations available are from optical [9] and Raman $[10,11]$ measurements performed in a laser heated diamond anvil cell. Interestingly, these studies all found a maximum on the melting curve although they disagree on its location: $50 \mathrm{GPa}$ for Ref. [9] and $70 \mathrm{GPa}$ for Refs. [10,11]. It is tempting to relate this observation of the melting curve maximum to the phase transition predicted by density functional theory between a low-density molecular liquid and a high-density polymeric and metallic liquid. Indeed, Ref. [8] showed that this liquid-liquid transition induces a maximum on the calculated melting line near $90 \mathrm{GPa}$. However, neither of the two experimental Raman studies found evidence of polymeric nitrogen in the fluid above the maximum melting temperature. Hence, the origin of the melting curve maximum remains unknown.

The existence of a polymeric liquid would be unambiguously evidenced by structure factor measurements. So far this information is limited on fluid nitrogen to $2.5 \mathrm{GPa}$ at ambient temperature [12]. Extending the structural investigation into the megabar range and under a few thousand kelvin temperature represents a formidable technical challenge. In this Letter, we report structural data on fluid nitrogen along the melting curve up to $122 \mathrm{GPa}$. This was made possible by using recently implemented $\mathrm{x}$-ray diffraction methods [13] coupled to advanced sample fabrication techniques. By contrast with previous works, we find a monotonic increase of the melting temperature up to $116 \mathrm{GPa}$ and $2080 \mathrm{~K}$, followed by a stiff melting slope increase due to the stability of the polymeric cg-N phase. Also, the evolution of the structure factor of liquid nitrogen demonstrates the persistence of the molecular liquid phase up to at least $120 \mathrm{GPa}$. Surprisingly, the $\mathrm{N} \equiv \mathrm{N}$ triple bond is more stable in the fluid than in the solid along melting.

The breakthrough that made possible the data collection of liquid nitrogen structure factors above $100 \mathrm{GPa}$ was the ability to stabilize, with local infrared laser heating in a diamond anvil cell (DAC), a homogeneous fluid volume on the time scale of minutes. Since nitrogen is optically transparent to the laser radiation, the use of an absorbing material is needed to heat the sample by thermal conduction. This absorber should not produce a large x-ray diffraction signal nor react with nitrogen at high pressure and temperature. The sample assembly is shown in Fig. 1. The nitrogen volume under study is sandwiched between 


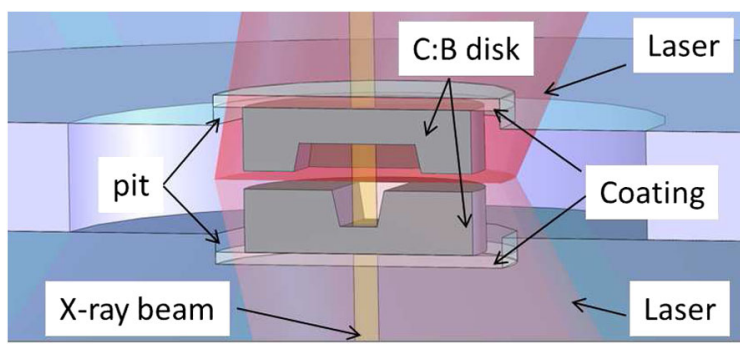

FIG. 1. Drawing of a cross-sectional view of the sample cavity located between the two diamond anvils of the DAC. The part of the sample probed by the $\mathrm{X}$-ray beam is at the intersection of the two slits engraved on the $\mathrm{C}: \mathrm{B}$ disks. The two disks form a capsule heated from both sides by yttrium lithium fluoride (YLF) laser beams. The laser focus was adjusted in order to fully illuminate the $\mathrm{C}: \mathrm{B}$ plates and reduce the radial temperature gradient.

two boron doped diamond $(\mathrm{C}: \mathrm{B})$ plates (diameter and thickness of $30-40 \mu \mathrm{m}$ and $10 \mu \mathrm{m}$, respectively). Blind rectangular slits of dimensions $10-15 \times 5 \mu \mathrm{m}^{2}$ and depth $5-6 \mu \mathrm{m}$ were machined at the center of the C:B plates using a focused ion beam machine to allow space for the nitrogen sample. When the sample is loaded, the two plates thus define a microcapsule enclosing the nitrogen sample probed by the x-ray beam. To ensure a good and steady positioning of the $\mathrm{C}: \mathrm{B}$ disks and increase the sample thickness, a pit of 40 to $50 \mu \mathrm{m}$ in diameter and up to $6 \mu \mathrm{m}$ in depth was machined by the focused ion beam at the center of the culet of the diamond anvils. The diamond anvils were coated with a $1-2 \mu \mathrm{m}$ layer of $\mathrm{Al}_{2} \mathrm{O}_{3}$ or $\mathrm{LiF}$ for thermal insulation. Finally, a micrograin of gold was placed on the side of the sample chamber to estimate the pressure from its measured volume. A rhenium foil was used as the gasket material. This sample assembly was loaded with $\mathrm{N}_{2}$ gas (99.9\% purity) under 1400 bar pressure. A total of five samples were studied in DACs equipped with diamond anvils of culet size ranging from 100 to $300 \mu \mathrm{m}$.

$\mathrm{X}$-ray diffraction measurements were performed on the ID27 beam line of the European Synchrotron Radiation Facility (ESRF). The x-ray beam was focused down to $3 \mu \mathrm{m}$ and aligned to coincide with the center of the C:B disks. The X-ray wavelength was $0.3738 \AA$ and the diffraction signal was collected on a Mar345 Image Plate or a Mar CCD detector. In order to extract the liquid nitrogen diffraction signal, which, for such a low- $Z$ system, is much weaker than the Compton scattering from the diamond anvils, an oscillating multichannel collimator (MCC) device was used [13]. The usefulness of this device for liquid structure factor measurements in a DAC has been recently demonstrated on fluid hydrogen up to $5 \mathrm{GPa}$ [14] and fluid $\mathrm{CO}_{2}$ up to $10 \mathrm{GPa}$ [15].

Samples were laser heated by two YLF lasers entering the DAC from both sides in order to reduce axial temperature gradients. The temperature was measured from both sides by analyzing the thermal radiation emitted by a $3 \mu \mathrm{m}$ diameter area at the center of the boron doped diamond disks and collected through reflective optics. The temperature homogeneity was also checked by measuring the temperature at various positions on the $\mathrm{C}: \mathrm{B}$ disks. The temperature was determined by a fit to the Planck distribution of the thermal emission spectrum recorded between 550 and $950 \mathrm{~nm}$. The uncertainty was estimated to be less than $100 \mathrm{~K}$ from the dispersion of the two-color temperature [16]. Samples were compressed at high temperature to maintain hydrostatic conditions. The pressure was determined using both the equations of state of gold [17] and diamond [18] (the compression curve of boron doped diamond is identical to the one of diamond within experimental uncertainty), which agreed within $2 \mathrm{GPa}$. A maximum thermal pressure of $4 \mathrm{GPa}$ could be observed at $100 \mathrm{GPa}$ and $2000 \mathrm{~K}$.

A total of 24 melting points were obtained between 40 and $125 \mathrm{GPa}$, with excellent reproducibility over the five experiments (see Fig. 2). The melting criterion was the appearance (disappearance) during the temperature increase (decrease) of a diffuse scattering ring in the $\mathrm{x}$-ray pattern coming from the liquid part of the sample. The diffuse rings appear more clearly after subtracting the x-ray pattern collected $200 \mathrm{~K}$ below the melting temperature, as shown in Fig. 2(b), since that removes the Compton contribution as well as the diffraction lines coming from the $\mathrm{C}: \mathrm{B}$ capsule and the $\left(\mathrm{LiF}\right.$ or $\mathrm{Al}_{2} \mathrm{O}_{3}$ ) coating. Above the melting temperature, the liquid signal increases with temperature as a consequence of the growing fraction of melted sample inside the capsule and reaches a maximum at a temperature $\sim 150 \mathrm{~K}$ above melting (this temperature interval can be taken as the temperature gradient inside the capsule). The melting temperature was defined as the lowest temperature for which the liquid signal was observed. Identical results were obtained upon increasing or decreasing the temperature. The complete set of investigated $P-T$ points is plotted in Fig. 2(c). A monotonic evolution of the melting temperature with pressure is observed up to $116 \mathrm{GPa}$ and the melting points are well fitted by a Simon and Glatzel equation [19] of the form $T_{m}=T_{0}\left(1+\Delta P_{m} / a\right)^{b}$, where $T_{0}=$ $585 \mathrm{~K}$ is the melting temperature at $P_{0}=10 \mathrm{GPa}$ [20], $\Delta P_{m}=P_{m}-P_{0}, a=3.0816 \mathrm{GPa}$, and $b=0.3553$.

Because of the small angular aperture of the MCC and the sample recrystallization into a few grains, the diffraction patterns of the solid phases, close to the melting curve, are incomplete. Yet, the observed reflections could be indexed in the cubic lattice of the $\delta$ - $\mathrm{N}_{2}$ phase up to $97 \mathrm{GPa}$ and in the rhombohedral lattice of the $\varepsilon-\mathrm{N}_{2}$ phase above. No cusp on the melting line is observed at the $\delta$ - $\epsilon$ transition, which is consistent with the very small volume difference between the two molecular phases inferred from ab initio simulations [8]. By contrast, a stiff increase in the slope of the melting curve occurs at the triple point joining the $\varepsilon-\mathrm{N}_{2}, \mathrm{cg}-\mathrm{N}$, and fluid phase at $116 \mathrm{GPa}$, $2080 \mathrm{~K}$. The two melting points above $116 \mathrm{GPa}$ were 


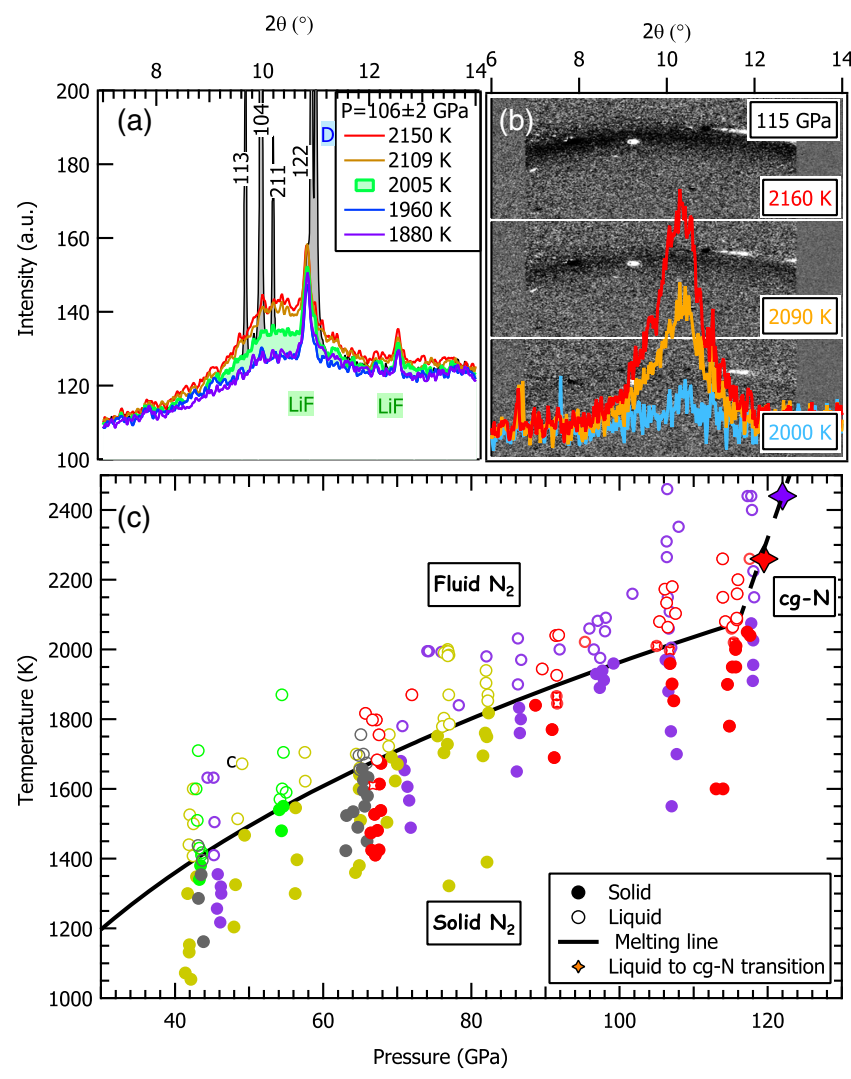

FIG. 2. Determination of the nitrogen melting curve from five experiments. (a) X-ray diffraction patterns collected at several temperatures around melting at $106 \mathrm{GPa}$. Solid nitrogen $\left(\varepsilon-\mathrm{N}_{2}\right)$, diamond, and $\mathrm{LiF}$ diffraction peaks are labeled. The broad diffuse ring centered at $2 \theta=10.5^{\circ}$ at $2005 \mathrm{~K}$ and above comes from melted nitrogen. (b) Background-subtracted x-ray diffraction 2D images collected at several temperatures around melting at $115 \mathrm{GPa}$ and the corresponding integrated patterns. (c) Pressuretemperature conditions at which $\mathrm{x}$-ray diffraction patterns have been collected. Each color represents a different sample. The solid (empty) circles represent the solid (liquid) phase. The lozenges indicate the $P-T$ conditions at which the cg-N melting is crossed during the pressure increase. The black line is a fit of the Kechin equation to the melting points (see text).

obtained upon isothermal compression of the fluid at 2260 and $2440 \mathrm{~K}$ in two separate samples. The fluid crystallized into the $\mathrm{cg}-\mathrm{N}$ phase, as revealed by the diffraction patterns, at respectively 119 and $122 \mathrm{GPa}$. The solidification was followed by a sharp pressure drop of $\sim 10 \mathrm{GPa}$, indicating a large volume discontinuity. We note that the $\mathrm{C}: \mathrm{B}$ disks were severely damaged at the transition due to the volume collapse, which prevented further uniform heating of the sample and thus terminated the data collection.

As shown in Fig. 3, the present finding of a monotonic increase of the nitrogen melting temperature contradicts previous claims reporting a downturn of the melting temperature $T_{m}$. First, Mukherjee and Boehler found a sharp maximum at $50 \mathrm{GPa}, 1920 \mathrm{~K}$ [9]. Melting detection

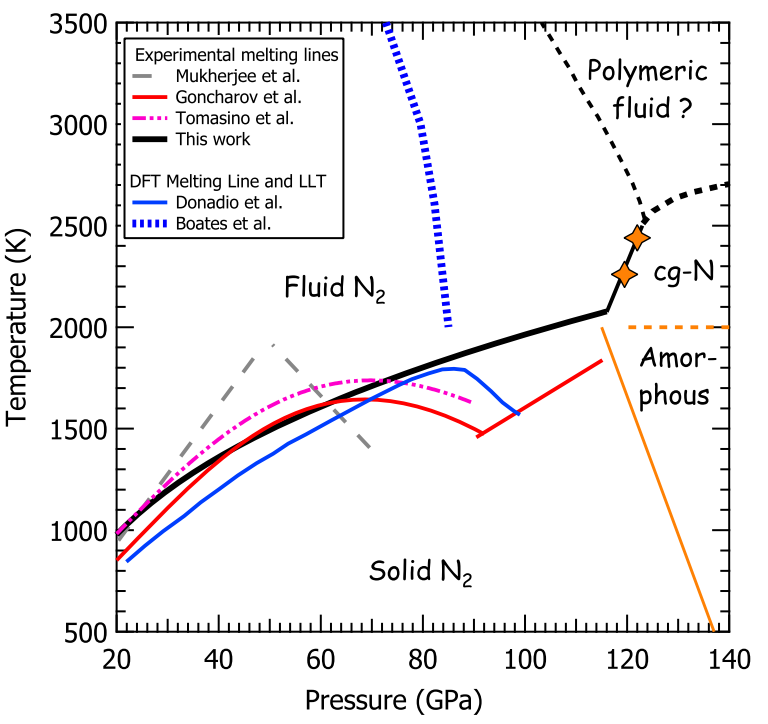

FIG. 3. Revisited phase diagram of nitrogen. The present determination of the melting curve is shown as a black line. Previous determinations from experiments [9-11] and from simulations [8] are shown as various color lines for comparison. The calculations of the low density liquid to high density liquid transition line (LLT) [7] are also presented as dashed lines. The $\delta$ - $\epsilon$ transition line is omitted for clarity.

relied on the visual observation of the speckle motion produced by illuminating the sample with a blue laser. However, this speckle motion became increasingly difficult to detect above $45 \mathrm{GPa}$ and impossible to detect above $70 \mathrm{GPa}$. Two other laser heating investigations of melting, carried out by Goncharov et al. [10] and by Tomasino et al. [11], were based on the inspection of the changes in the vibrational Raman spectrum. These two melting curves are similar, except for a global $120 \mathrm{~K}$ temperature shift likely due to different temperature measurements, and display a progressive downturn and a maximum $T_{m}$ of $\sim 1700 \mathrm{~K}$ at $70 \mathrm{GPa}$. Our measurements agree well with Refs. [10,11] up to $60 \mathrm{GPa}$ but at $90 \mathrm{GPa}$ the temperature differs by $280-400 \mathrm{~K}$, which is larger than the expected error on the temperature measurements. We believe this discrepancy originates from the difficulties in clearly detecting melting above $1600 \mathrm{~K}$ in Refs. [10,11], which are absent in the present work. Raman measurements are perturbed by the thermally induced radiation in the visible range, which strongly rises with temperature, making the detection of Raman modes increasingly difficult, as attested by the Raman spectra reported in Ref. [10]. This is not the case for $\mathrm{x}$-ray diffraction. Moreover, in our sample geometry, the volume of the nitrogen sample seen by the x-ray beam does not change much over the pressure range; thus, the sample to background signal ratio is nearly constant with pressure and temperature. The temperature homogeneity of the probed sample is also improved in the present work thanks to the use of microcapsules heated from both sides. This very homogeneous heating and the performance of our 
detection method are also demonstrated by the small temperature steps, typically less than $100 \mathrm{~K}$, separating $\mathrm{x}$-ray patterns with a liquid signal from those with solely solid diffraction.

A phase transition from the molecular $\mathrm{N}_{2}$ fluid to a metallic polymeric liquid has been predicted by ab initio molecular dynamics simulations at about $88 \mathrm{GPa}[7,8,21]$ (see Fig. 3). At the transition, polymeric chains of singly or doubly bonded $\mathrm{N}$ atoms are formed at the expense of triply bonded molecules whose number decreases to zero by $120 \mathrm{GPa}$. The Raman experiments of Refs. [10,11] did not find evidence of $\mathrm{N}=\mathrm{N}$ and/or $\mathrm{N}-\mathrm{N}$ vibrations or of an intensity drop of the molecular vibron in the fluid above $90 \mathrm{GPa}$. The authors argued that the change in the Raman spectra at the transition could be too "subtle" to be observed. In the present study, a clear x-ray diffraction signal of the molten state is observed and analyzed in terms of the liquid structure factor $S(Q)$. The evolution of $S(Q)$ along the melting line is shown at various pressures in Fig. 4. The signal to background ratio is much better for $S(Q)$ at $4.5 \mathrm{GPa}$ since measurements could in that case be done in a resistively heated DAC on a much larger liquid volume. $S(Q)$ at $4.5 \mathrm{GPa}$ can thus be taken as a reference structure factor of molecular $\mathrm{N}_{2}$ fluid. A main diffraction peak (MDP) is observed followed by very weak oscillations, similarly to previous measurements [12]. As seen in Fig. 4, a shift of the MDP is clearly observed with pressure but the shape of $S(Q)$ does not change up to the highest pressure. The calculated modifications of $S(Q)$ associated with the transition from the molecular fluid to the polymeric liquid are not observed. The computed structure factor of the polymeric liquid differs from that of the molecular liquid by a drop in intensity of the MDP and the emergence of a second peak around $6 \AA^{-1}$ of similar intensity as the MDP [7]. Also, the position of the MDP $\left(Q_{m}\right)$ should shift more slowly with pressure in the polymeric liquid since the latter is less compressible than the molecular one [7], but no such trend is observed in our data [see Fig. 4(b)]. In the insert of Fig. 4(b), we have tried to quantify whether fluid nitrogen behaves as a simple molecular fluid and so we have plotted $Q_{m}^{3}$ versus density [22]. A linear relation between $Q_{m}^{3}$ and density is observed, as expected for a simple liquid [24], over the whole fluid domain measured here. We can thus confidently state that our x-ray data show that the molecular $\mathrm{N}_{2}$ fluid is stable along the melting line up to $116 \mathrm{GPa}$.

Three concluding remarks can be made. First, ab initio calculations $[7,8,21]$ seem to underestimate the stability range of the molecular fluid phase. These theoretical studies all used the PBE-GGA approximation for the exchange-correlation functional without taking into account dispersive interactions. However, it was recently shown that the effect of van der Waals interactions is to widen the thermodynamical domain of stability of the
$Q(\AA)$


FIG. 4. Structural evolution of fluid nitrogen. (a) Experimental structure factors of fluid nitrogen collected at various $P-T$ conditions along the melting line. The structure factor obtained at $4.5 \mathrm{GPa}$ in a distinct experiment under resistive heating is shown for comparison. (b) Evolution of the position $\left(Q_{m}\right)$ of the main diffraction peak with pressure. Inset: $\left(Q_{m} / Q_{m_{0}}\right)^{3}$ plotted against $\rho / \rho_{0}$, where $\rho$ is the fluid density computed by a classical molecular dynamics simulation (see text). The dashed lines are a guide for the eyes.

molecular phase [25]. This advanced level of theoretical description seems therefore needed to compute the high temperature nitrogen phase diagram. Second, contrary to the current views, we show that polymeric nitrogen occurs at a higher pressure in the fluid than in the solid phase. This suggests that entropic effects favor the molecular phase over the extended covalent one, in line with recent predictions [25]. An accurate description of the phase diagram of hot dense nitrogen will thus require an accurate treatment of entropic terms. Third, we show here that no polymeric liquid exists for $T<2440 \mathrm{~K}$ but it may be conjectured that the polymeric liquid exists at higher $T$, beyond a line that connects the fully dissociated fluid around $30 \mathrm{GPa}$ and $\sim 6000 \mathrm{~K}$ [5] to the polymeric solid above $120 \mathrm{GPa}$ and $2500 \mathrm{~K}$ (see Fig. 3). Determining this transition line is certainly a challenge for future experiments and a benchmark for theory. 
We acknowledge the European Synchrotron Radiation Facility for the provision of beam time under the auspices of the long-term project HD-463 and run HC2490, and financial support from the Agence Nationale de la Recherche under Grant No. ANR-13-BS04-0015 (MOFLEX). We thank Johann Bouchet for discussions, Olivier Marie for the focused ion beam work and Stany Bauchau for his help with the $\mathrm{x}$-ray experiment design.

[1] A. F. Goncharov, E. Gregoryanz, H. K. Mao, Z. Liu, and R. J. Hemley, Phys. Rev. Lett. 85, 1262 (2000).

[2] M. I. Eremets, R. J. Hemley, H. K. Mao, and E. Gregoryanz, Nature (London) 411, 170 (2001).

[3] M. I. Eremets, A. G. Gavriliuk, N. R. Serebryanaya, I. A. Trojan, D. A. Dzivenko, R. Boehler, H. K. Mao, and R. J. Hemley, J. Chem. Phys. 121, 11296 (2004).

[4] D. Tomasino, M. Kim, J. Smith, and C.-S. Yoo, Phys. Rev. Lett. 113, 205502 (2014).

[5] W. J. Nellis, N. C. Holmes, A. C. Mitchell, and M. van Thiel, Phys. Rev. Lett. 53, 1661 (1984).

[6] R. Chau, A. C. Mitchell, R. W. Minich, and W. J. Nellis, Phys. Rev. Lett. 90, 245501 (2003).

[7] B. Boates and S. A. Bonev, Phys. Rev. Lett. 102, 015701 (2009).

[8] D. Donadio, L. Spanu, I. Duchemin, F. Gygi, and G. Galli, Phys. Rev. B 82, 020102 (2010).

[9] G. D. Mukherjee and R. Boehler, Phys. Rev. Lett. 99, 225701 (2007).

[10] A. F. Goncharov, J. C. Crowhurst, V. V. Struzhkin, and R. J. Hemley, Phys. Rev. Lett. 101, 095502 (2008).

[11] D. Tomasino, Z. Jenei, W. Evans, and C. S. Yoo, J. Chem. Phys. 140, 244510 (2014).
[12] L. Temleitner, L. Pusztai, Y. Akahama, H. Kawamura, S. Kohara, Y. Ohishi, and M. Takata, Phys. Rev. B 78, 014205 (2008).

[13] G. Weck, G. Garbarino, S. Ninet, D. Spaulding, F. Datchi, P. Loubeyre, and M. Mezouar, Rev. Sci. Instrum. 84, 063901 (2013).

[14] G. Weck, G. Garbarino, P. Loubeyre, F. Datchi, T. Plisson, and M. Mezouar, Phys. Rev. B 91, 180204(R) (2015).

[15] F. Datchi, G. Weck, A. M. Saitta, Z. Raza, G. Garbarino, S. Ninet, D. K. Spaulding, J. A. Queyroux, and M. Mezouar, Phys. Rev. B 94, 014201 (2016).

[16] L. R. Benedetti and P. Loubeyre, High Press. Res. 24, 423 (2004).

[17] K. Takemura and A. Dewaele, Phys. Rev. B 78, 104119 (2008).

[18] A. Dewaele, F. Datchi, P. Loubeyre, and M. Mezouar, Phys. Rev. B 77, 094106 (2008).

[19] F. Simon and G. Glatzel, Z. Anorg. Allg. Chem. 178, 309 (1929).

[20] A. S. Zinn, D. Schiferl, and M. F. Nicol, J. Chem. Phys. 87, 1267 (1987).

[21] K. P. Driver and B. Militzer, Phys. Rev. B 93, 064101 (2016).

[22] The density of the fluid was estimated by classical molecular dynamics simulations using the effective pair potential fitted on the pressure-compression data of the shocked liquid [23]. The parameters of the Buckingham potential used here are $\alpha=11.5, \varepsilon=293 \mathrm{~K}$, and $r^{\star}=3.91 \AA$.

[23] M. van Thiel and F. H. Ree, J. Chem. Phys. 104, 5019 (1996).

[24] A. B. Bhatia and N. H. March, Phys. Chem. Liq. 13, 313 (1984).

[25] A. Erba, L. Maschio, C. Pisani, and S. Casassa, Phys. Rev. B 84, 012101 (2011). 